\title{
FOLIATIONS TRANSVERSE TO FIBERS OF A BUNDLE
}

\author{
J. F. PLANTE
}

\begin{abstract}
Consider a fiber bundle where the base space and total space are compact, connected, oriented smooth manifolds and the projection map is smooth. It is shown that if the fiber is null-homologous in the total space, then the existence of a foliation of the total space which is transverse to each fiber and such that each leaf has the same dimension as the base implies that the fundamental group of the base space has exponential growth.
\end{abstract}

Introduction. Let $(E, p, B)$ be a locally trivial fiber bundle where $p: E \rightarrow B$ is the projection, $E$ and $B$ are compact, connected, oriented smooth manifolds and $p$ is a smooth map. (By smooth we mean $C^{r}$ for some $r \geqq 1$ and, henceforth, all maps are assumed smooth.) Let $b$ denote the dimension of $B$ and $k$ denote the dimension of the fiber (thus, $\operatorname{dim} E=$ $b+k$ ). By a section of the fibration we mean a smooth map $\sigma: B \rightarrow E$ such that $p \circ \sigma=\mathrm{id}_{B}$. It is well known that if a section exists then the fiber over any point in $B$ represents a nontrivial element in $H_{k}(E ; Z)$ since the image of a section is a compact orientable manifold which has intersection number one with the fiber over any point in $B$. The notion of a section may be generalized as follows.

Definition. A polysection of $(E, p, B)$ is a covering projection $\pi: \widetilde{B} \rightarrow B$ together with a map $\xi: \widetilde{B} \rightarrow E$ such that the following diagram commutes.

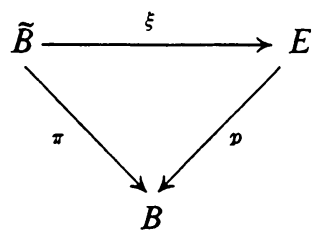

Note that if the covering projection is a diffeomorphism then the map $\xi \circ \pi^{-1}$ is a section in the usual sense.

One important situation in which polysections arise is the following. Suppose that $\mathscr{F}$ is a smooth foliation of $E$ such that each leaf of $\mathscr{F}$ is

Received by the editors April 4, 1973 and, in revised form, May 1, 1973.

AMS (MOS) subject classifications (1970). Primary 57D30; Secondary 55F10.

Key words and phrases. Foliation, fiber bundle, section, intersection number, covering projection, growth function, asymptotic homology class.

(c) American Mathematical Society 1974 
transverse to the fibers and has the same dimension as $B$. In such a situation, a well-known theorem of Ehresmann says that the restriction of $p$ to each leaf $L$ of $\mathscr{F}$ is a covering projection and, thus, the inclusion map $i: L \rightarrow E$ represents a polysection of $(E, p, B)$. The problem of finding which circle bundles over compact surfaces admit such transverse foliations has been completely solved by J. Wood [8]. Theorem 1 below implies a partial result for higher dimensional bundles.

Statement of results. Before stating results it is necessary to recall certain definitions and facts concerned with the notion of growth [3], [5].

Definition. Let $M$ be a connected Riemannian manifold and let $x \in M$. The growth function at $x$ is the continuous increasing function $g: \boldsymbol{R}^{+} \rightarrow \boldsymbol{R}^{+}$defined by

$$
g(t)=\operatorname{vol}\{y \in M \mid \operatorname{dist}(x, y) \leqq t\} .
$$

$M$ has exponential growth if there exist positive numbers $A, \alpha$ such that for sufficiently large $t, g(t) \geqq A \exp \alpha t$. (Note that this last concept is independent of the point $x$ which is chosen.)

Definition. Let $\Gamma$ be a finitely generated discrete group with a fixed finite generating set $\mathscr{S}$. For $\gamma \in \Gamma$ let $m(\gamma)$ denote the minimum possible word length that $\gamma$ can have (with respect to the generating set $\mathscr{S}$ ). The growth function of $(\Gamma, \mathscr{S})$ is the increasing function $g: Z^{+} \rightarrow Z^{+}$defined by

$$
g(n)=\text { number of elements } \gamma \in \Gamma \text { such that } m(\gamma) \leqq n .
$$

$\Gamma$ has exponential growth if for some $A, \alpha>0, g(n) \geqq A \exp \alpha n$. (This last concept is independent of the generating set $\mathscr{S}$.)

Proposition ([3], [5]). If $M$ is a compact connected Riemannian manifold then the universal covering space of $M$ (with induced metric) has exponential growth iff $\pi_{1}(M)$ has exponential growth.

We assume from now on that the manifolds $E$ and $B$ of our fibration have fixed Riemannian metrics. It will be noted, however, that because of the compactness the results stated below are independent of the Riemannian metrics chosen. Also any covering space of the Riemannian manifold $B$ will be assumed to have the Riemannian metric induced by the covering projection.

Definition. A polysection $\pi: \widetilde{B} \rightarrow B, \xi: \widetilde{B} \rightarrow E$ is bounded if the derivative of $\xi$ has bounded norm.

As above, this definition is independent of the Riemannian metrics chosen for $B$ and $E$. Also note that polysections which arise from foliations transverse to the fibers of $E$ are bounded. 
THEOREM 1. If $\pi: \widetilde{B} \rightarrow B, \xi: \widetilde{B} \rightarrow E$ is a bounded polysection of the bundle $(E, p, B)$ and if the fiber over some point in $B$ is homologous to zero in $H_{k}(E ; R)$ then $\widetilde{B}$ has exponential growth.

In particular the conclusion of Theorem 1 together with the proposition stated above imply that $\pi_{1}(B)$ has exponential growth. Thus, we have

COROLlARY 1. If $\pi_{1}(B)$ does not have exponential growth and some fiber is null-homologous in $H_{k}(E ; R)$ then the bundle $(E, p, B)$ does not have any bounded polysections.

Corollary 2. If $(E, p, B)$ is as in Corollary 1 then there is no foliation transverse to the fibers with leaves having the same dimension as $B$.

The proof of Theorem 1 is given in the next section.

REMARK ([7]). Finitely generated groups which do not have exponential growth include all finite extensions of nilpotent groups.

EXAMPLE. When the fiber is $S^{k}$ we have from the Gysin cohomology sequence [6] (real coefficients)

$$
H^{k}(B) \stackrel{p *}{\longrightarrow} H^{k}(E) \longrightarrow H^{0}(B) \stackrel{\Psi}{\longrightarrow} H^{k+1}(B)
$$

where $\Psi(1) \in H^{k+1}(B)$ is just the Euler class of the bundle. Hence, the fiber is null-homologous in $H_{k}(E)$ iff $p^{*}$ is surjective which is true iff the Euler class is nonzero. Thus, a sphere bundle with nonzero Euler class and a foliation transverse to the fibers has the property that $\pi_{1}(B)$ has exponential growth. In particular, consider the case where $B$ is a compact surface of negative curvature and $(E, p, B)$ is its unit tangent bundle. By the Gauss-Bonnet theorem the Euler class is nonzero and there exist foliations which are transverse to the fibers [1]. Thus, we retrieve a special case of Milnor's result [3] which says that $\pi_{1}(B)$ has exponential growth, whenever $B$ is a compact Riemannian manifold with negative sectional curvatures.

Proof of Theorem 1. Suppose that $\xi: \widetilde{B} \rightarrow E$ is a polysection of $(E, p, B)$ and assume that $\widetilde{B}$ does not have exponential growth. We will show that the fiber must represent a nonzero element in $H_{k}(E ; R)$. Our argument will be analogous to the intersection number argument used for sections except that we use the notion of an asymptotic homology class as introduced in [4].

Definition. Let $M$ be a compact Riemannian manifold and let $N_{i}$ $(i=1,2, \cdots)$ be a sequence of compact submanifolds of $M$ such that

(1) dimension $N_{i}=q$ for all $i$.

(2) int $N_{i}$ is a smooth submanifold of $M$ and $\partial N_{i}$ is piecewise-smooth. 
(3) $\lim _{i \rightarrow \infty}\left(\operatorname{vol} \partial N_{i} / \operatorname{vol} N_{i}\right)=0$, where the volumes are determined by restricting the Riemannian metric on $M$ to the submanifolds $N_{i}$ and $\partial N_{i}$.

(4) There exist closed forms $\eta_{1}, \cdots, \eta_{l}$ generating $H^{q}(M ; R)$ such that

exists for $j=1, \cdots, l$.

$$
\lim _{i \rightarrow \infty} \frac{1}{\operatorname{vol} N_{i}} \int_{N_{i}} \eta_{j}
$$

The asymptotic homology class $A \in H_{q}(M ; R)$ of the sequence $N_{i}$ is the map $A: H^{q}(M ; \boldsymbol{R}) \rightarrow \boldsymbol{R}$ defined by

$$
A(\eta)=\lim _{i \rightarrow \infty} \frac{1}{\operatorname{vol} N_{i}} \int_{N_{i}} \eta
$$

where $\eta$ is a closed $q$-form on $M$. (Here we think of $H_{q}(M ; R)$ as being the dual of $H^{q}(M ; R)$.) Conditions (3) and (4) together with Stokes' theorem insure that $A$ is well defined.

We return now to the proof of Theorem 1 .

By taking a triangulation of $B$ and lifting it upstairs to $\widetilde{B}$ we can construct a collection of fundamental domains (with piecewise-smooth boundaries) which cover $\widetilde{B}$ and intersect only along boundaries. Let $\tilde{x}$ be a fixed basepoint for $\widetilde{B}$ which is interior to some fundamental domain. For $t>0$ let $\Delta_{t}$ be the union of all fundamental domains which are within distance $t$ of $\tilde{x}$. Since we are assuming that $\widetilde{B}$ does not have exponential growth we have

$$
\lim _{t \rightarrow \infty} \inf \frac{\operatorname{vol} \partial \Delta_{t}}{\operatorname{vol} \Delta_{t}}=0
$$

By taking subsequences (so as to satisfy (3) and (4) above) we obtain a sequence $t_{i} \rightarrow \infty$ such that the sequence of submanifolds $N_{i}=\xi\left(\Delta_{t_{i}}\right)$ defines an asymptotic homology class $A \in H_{b}(E ; R)$. Roughly speaking the proof will be completed by showing that $\xi(\widetilde{B})$ has nonzero "asymptotic intersection number" with a fiber. To do this let $x=p(\tilde{x})$ and let $f: B \rightarrow \boldsymbol{R}$ be a smooth (bump) function which is nonnegative and has (nonempty) support in an evenly covered (with respect to $\pi: \widetilde{B} \rightarrow B$ ) neighborhood. Now let $\Omega$ be thc Riemannian volume form on $B . f \Omega$ is a closed form and, therefore, so is $p^{*}(f \Omega)$. The proof will be completed by showing that $A\left(p^{*}(f \Omega)\right)>0$. By boundedness of $\xi$ there exists $\lambda<\infty$ such that vol $\xi(D) \leqq$ $\lambda$ for any fundamental domain $D$ (where vol $\xi(D)$ refers to the volume as a submanifold of $E$ ). Since the integral of $p^{*}(f \Omega)$ over the image under $\xi$ of a fundamental domain is equal to $\int_{B} f \Omega$ we have

$$
A\left(p^{*}(f \Omega)\right)=\lim _{i \rightarrow \infty} \frac{1}{\operatorname{vol} N_{i}} \int_{N_{i}} p^{*}(f \Omega) \geqq \frac{1}{\lambda} \int_{B} f \Omega>0 .
$$

This completes the proof of Theorem 1. 
Remark. This approach also yields a generalization of Theorem 1 of [4]. Namely, if a minimal set of a codimension $k$ foliation intersects an oriented compact $k$-dimensional manifold (with trivial normal bundle) which is transverse to the foliation then every leaf of the minimal set has exponential growth. This fact has been observed independently by $\mathrm{W}$. Thurston.

\section{REFERENCES}

1. D. V. Anosov, Geodesic flows on closed Riemannian manifolds of negative curvature, Trudy Mat. Inst. Steklov. 90 (1967)=Proc. Steklov Inst. Math. 90 (1967). MR 36 \#7157; 39 \#3527.

2. S. S. Chern, A simple intrinsic proof of the Gauss-Bonnet formula for closed Riemannian manifolds, Ann. of Math. (2) 45 (1944), 747-752. MR 6, 106.

3. J. Milnor, A note on curvature and the fundamental group, J. Differential Geometry 2 (1968), 1-7.. MR 38 \#636.

4. J. F. Plante, A generalization of the Poincaré-Bendixson theorem for foliations of codimension one, Topology 12 (1973), 177-181.

5. - Asymptotic properties of foliations, Comment. Math. Helv. 47 (1972), 449-456.

6. E. H. Spanier, Algebraic topology, McGraw-Hill, New York, 1966. MR 35 \#1007.

7. J. Wolf, Growth of finitely generated solvable groups and curvature of Riemannian manifolds, J. Differential Geometry 2 (1968), 421-446. MR 40 \#1939.

8. J. W. Wood, Bundles with totally disconnected structure groups, Comment. Math. Helv. 46 (1971), 257-273. MR 45 \#2732.

Department of Mathematics, University of Wisconsin, Madison, Wisconsin 53706

Current address: Department of Mathematics, University of North Carolina, Chapel Hill, North Carolina 27514 